\section{Mycobacterium leprae on Palatine Tonsils and Adenoids of Asymptomatic Patients, Brazil}

\author{
Marilda Aparecida Milanez Morgado de Abreu, ${ }^{1}$ \\ Gisele Alborghetti Nai, Juliana D'Andrea Molina, \\ Rafael Tomaz Gomes, Natalia de Paula, \\ Ana Maria Roselino ${ }^{1}$
}

Author affiliations: Regional Hospital, Presidente Prudente, Brazil (M.A.M. Morgado de Abreu, R.T. Gomes); University of Oeste Paulista, Presidente Prudente (M.A.M. Morgado de Abreu, G.A. Nai, J.A. Molina); and University of São Paulo, Ribeirão Preto, Brazil (N. de Paula, A.M. Roselino).

DOI: https://doi.org/10.3201/eid2610.191267

We investigated palatine tonsil and adenoid specimens excised from otorhinolaryngological patients in a leprosyendemic region of Brazil. Fite-Faraco staining identified Mycobacterium spp. in 9 of 397 specimen blocks. Immunohistochemistry and molecular analysis confirmed the presence of Mycobacterium leprae, indicating that these organs can house $M$. leprae in persons inhabiting a leprosy-endemic region.

L eprosy is a chronic infectious disease caused by Mycobacterium leprae that especially affects skin and peripheral nerves (1). In 2018, the registered global prevalence in the 6 World Health Organization regions was 184,238 cases $(0.24 / 10,000$ population), showing a decrease of 8,475 cases over the previous year (2). Although its incidence in Brazil has declined during 2009-2018, leprosy continues to be a major public health problem at the national level (1). Reports of $M$. leprae resistance against antimicrobial drugs used in multidrug therapy raise concern about the future of leprosy treatment (2). Therefore, not only does leprosy persist, but the emergence of multidrug-resistant $M$. leprae is a potential threat to global public health $(3,4)$.

Although the exact mode of leprosy transmission is not known, it is thought that the upper respiratory tract, in particular the nasal mucosa, is the usual site of primary infection (3). Because we have previously identified $M$. leprae in oral mucosa of leprosy patients (5), we aimed to investigate other anatomic sites that could host this microorganism to clarify the epidemiology and transmission mechanisms of leprosy.

In this study, we hypothesized that M. leprae, after penetration through the airway mucosa, could

${ }^{1}$ These authors contributed equally to this article. infect the palatine tonsils and adenoids, because these organs represent the first immune defense line against inhaled or ingested antigens (6). We also theorized that if leprosy is a highly contagious disease (1), a considerable part of the population in endemic regions might be infected with $M$. leprae.

We conducted a cross-sectional study of 397 paraffin-embedded blocks of palatine tonsils and adenoids extracted from 144 patients due to otorhinolaryngological indication during 2011-2016 at the Regional Hospital, Presidente Prudente, Brazil. The local Research Ethics Committee approved the study (protocol \#1.920.994).

Microscopic analysis using hematoxylin-eosin staining did not reveal granulomas. We analyzed 50 fields in the $100 \times$ objective $(1,000 \times$ magnification) per slide stained with Fite-Faraco; of the positive cases (9 [2.3\%] slides from 8 [5.6\%] patients, 6 men and 2 women [mean age $11 \pm 5.5$ years]), we observed only 1 acid-fast rod per slide. We studied all the blocks of these 8 patients, a total of 20 blocks (Table).

Immunohistochemistry with 1:20,000 anti-phenolic glycolipid-I (anti-PGL-I) antibody (Bei Resources, https://www.beiresources.org), specific for $M$. leprae, was conducted with the Mach 1 polymer-based biotin-free detection kit (Biocare Medical, https:// biocare.net) (5). We used deparaffinized skin sections from multibacillary leprosy as positive control. For the negative control, we omitted the antibody. To confirm specificity, we used deparaffinized skin sections from paucibacillary leprosy and atopic dermatitis (excluding inflammatory cell recognition by the antibody), normal human scalp, and tuberculous lung section.

We extracted DNA from paraffin sections with isopropanol-ammonium acetate (1). The resulting DNA was used in conventional PCR with sense 5'-ATTTCTGCCGCTGGTATCGGT-3' and antisense 5'-TGCGCTAGAAGGTTGCCGTAT-3' primers (ThermoFisher Scientific, https:/ / www.thermofisher.com) to amplify $M$. leprae microsatellite sequences, according to a previous report (7). We assessed amplicons of $148 \mathrm{bp}$ on $2 \%$ agarose gel. The assays included negative (DNA omission) and positive (DNA from multibacillary leprosy skin sample) controls. We confirmed specificity with DNA extracted from $M$. tuberculosis culture. In addition, we conducted PCR with TB1/TB2 primers to detect Mycobacterium spp. and with T4/T5 primers to detect $M$. tuberculosis (5).

Immunohistochemistry was positive in $18 / 20$ (90\%) blocks. By PCR, 19 (95\%) were positive with RLEP and 5 were simultaneously positive with TB1/ TB2; all 19 positive by PCR were negative by T4/T5 
Table. Patient data and results of Fite-Faraco staining, immunohistochemistry with anti-PGL-I antibody, and PCR assays in study of Mycobacterium leprae on palatine tonsils and adenoids, Brazil, 2019*

\begin{tabular}{|c|c|c|c|c|c|c|}
\hline Patient no. & Age, y/sex & Lymphoid organ & Fite-Faraco stain & IHC anti-PGL-I & PCR RLEP & $\begin{array}{l}\text { M. leprae identification by } \\
\text { DNA sequencing, \% }\end{array}$ \\
\hline 1 & $20 / \mathrm{F}$ & $\mathrm{AD}$ & + & + & + & ND \\
\hline \multirow[t]{2}{*}{2} & 19/M & RPT & + & + & + & ND \\
\hline & & LPT & - & + & + & ND \\
\hline \multirow[t]{2}{*}{3} & 10/M & $\mathrm{RPT}$ & + & + & + & 100 \\
\hline & & LPT & - & + & + & ND \\
\hline \multirow[t]{3}{*}{4} & 9/M & RPT & - & + & + & ND \\
\hline & & LPT & + & + & + & 99 \\
\hline & & $\mathrm{AD}$ & + & + & + & ND \\
\hline \multirow[t]{3}{*}{5} & 7/M & RPT & - & + & + & ND \\
\hline & & LPT & + & + & + & ND \\
\hline & & $\mathrm{AD}$ & - & + & + & ND \\
\hline \multirow[t]{3}{*}{6} & 4/M & RPT & - & + & + & ND \\
\hline & & LPT & + & + & + & 99 \\
\hline & & $A D$ & - & - & + & ND \\
\hline \multirow[t]{3}{*}{7} & $13 / F$ & RPT & + & + & + & 98 \\
\hline & & LPT & - & + & + & ND \\
\hline & & $A D$ & - & + & + & ND \\
\hline \multirow[t]{3}{*}{8} & 6/M & RPT & - & - & - & ND \\
\hline & & LPT & + & + & + & ND \\
\hline & & $\mathrm{AD}$ & - & + & + & 98 \\
\hline \multicolumn{3}{|c|}{ Total positive results } & 9 & 18 & 19 & \\
\hline
\end{tabular}

primers. Samples that tested negative in IHC or PCR were also negative in Fite-Faraco staining (Table).

Little attention has been paid to the role that mucosa-associated lymphoid tissue (MALT) plays in the mechanisms used by mycobacteria during host invasion. In tuberculosis infection, bacilli cross mucous membranes and penetrate into palatine tonsils and adenoids, where they initiate an immune response. However, bacilli may develop immune-evasion strategies and disseminate into the organism or return to the mucosa surface and be eliminated to the environment (8). This process might also occur in leprosy, but to our knowledge there are no reports on this subject.

Our results corroborate the hypothesis that $M$. leprae bacilli infect palatine tonsils and adenoids. Prospective studies with a larger population group are necessary to clarify these findings. We could not infer from this retrospective study with paraffinized samples whether patients who had positive results for $M$. leprae identification had leprosy or were asymptomatic carriers. In both clinical scenarios, however, our findings indicate that palatine tonsils and adenoids may represent reservoirs for $M$. leprae bacilli in persons inhabiting a leprosy-endemic region.

\section{Acknowledgments}

We thank Sandra Rodrigues and Aline Turatti for their technical laboratory assistance.

The Paulista Foundation against Leprosy provided partial research support.

\section{About the Author}

Dr. Morgado de Abreu is a professor and coordinator at the program of specialization in dermatology, Regional Hospital and University of Oeste Paulista, Presidente Prudente. Her research interests include infectious diseases, with emphasis on the epidemiology and mechanisms of leprosy transmission.

\section{References}

1. Lastória JC, Abreu MA. Leprosy: review of the epidemiological, clinical, and etiopathogenic aspects - part 1. An Bras Dermatol. 2014;89:205-18. https://doi.org/10.1590/ abd1806-4841.20142450

2. World Health Organization. Global leprosy update, 2018: moving towards a leprosy-free world. WER No. 35/36. Wkly Epidemiol Rec. 2019;94:389-412.

3. Levis W, Rendini T, Martiniuk F. Increasing virulence in leprosy indicated by global Mycobacterium spp. Emerg Infect Dis. 2018;24:183a-184. https:// doi.org/10.3201/eid2401.171785

4. Cambau E, Saunderson P, Matsuoka M, Cole ST, Kai M, Suffys $\mathrm{P}$, et al.; WHO surveillance network of antimicrobial resistance in leprosy. Antimicrobial resistance in leprosy: results of the first prospective open survey conducted by a WHO surveillance network for the period 2009-15. Clin Microbiol Infect. 2018;24:1305-10. https://doi.org/ 10.1016/j.cmi.2018.02.022

5. Morgado de Abreu MAM, Roselino AM, Enokihara M, Nonogaki S, Prestes-Carneiro LE, Weckx LLM, et al. Mycobacterium leprae is identified in the oral mucosa from paucibacillary and multibacillary leprosy patients. Clin Microbiol Infect. 2014;20:59-64. https://doi.org/10.1111/ 1469-0691.12190

6. Brandtzaeg P. Immunology of tonsils and adenoids: everything the ENT surgeon needs to know. Int J Pediatr Otorhinolaryngol. 2003;67(Suppl 1):S69-76. https:/ / doi. org/10.1016/j.ijporl.2003.08.018 
7. Azevedo MC, Ramuno NM, Fachin LR, Tassa M, Rosa PS, Belone AF, et al. qPCR detection of Mycobacterium leprae in biopsies and slit skin smear of different leprosy clinical forms. Braz J Infect Dis. 2017;21:71-8. https:/ / doi.org/ 10.1016/j.bjid.2016.09.017

8. Lugton I. Mucosa-associated lymphoid tissues as sites for uptake, carriage and excretion of tubercle bacilli and other pathogenic mycobacteria. Immunol Cell Biol. 1999;77:364-72. https:/ / doi.org/10.1046/j.1440-1711.1999.00836.x

Address for correspondence: Marilda A.M. Morgado de Abreu, rua São Paulo, 1949, centro, CEP 17900-000, Dracena, SP, Brazil; email: marilda@morgadoeabreu.com.br

\section{Fatal Chlamydia avium Infection in Captive Picazuro Pigeons, the Netherlands}

\author{
Marja Kik, Marloes Heijne, Jooske IJzer, Guy \\ Grinwis, Yvonne Pannekoek, Andrea Gröne
}

Author affiliations: Utrecht University, Utrecht, the Netherlands (M. Kik, J. IJzer, G. Grinwis, A. Gröne); Wageningen Bioveterinary Research, Lelystad, the Netherlands (M. Heijne); University of Amsterdam, Amsterdam, the Netherlands (Y. Pannekoek)

DOI: https://doi.org/10.3201/eid2610.191412

In 2016, an outbreak of Chlamydia avium infection occurred among Picazuro pigeons (Patagioenas picazuro) living in an aviary in the Netherlands. Molecular typing revealed a unique strain of $C$. avium. Our findings show that $C$. avium infection, which usually causes subclinical infection, can cause fatal disease in pigeons.

Ut ntil approximately 2014, Chlamydia psittaci was the only Chlamydia species detected in birds. Researchers have catalogued $\approx 465$ bird species affected by this pathogen, which mainly causes subclinical infections but sometimes results in acute disease and death (1). In humans, C. psittaci is highly infectious and can cause severe pneumonia. Chlamydia bacteria, which are present in (dried) excreta or feather dust, are transmitted through direct contact or inhalation. In 2014, researchers proposed 2 new members of Chlamydiaceae: C. avium and C. gallinacea (2). C. avium affects pigeons and psittacine birds, whereas
C. gallinacea affects poultry. Most C. avium and C. gallinacea infections in birds are subclinical, and the zoonotic potential of these species is unknown (3).

In 2016, an outbreak of C. avium infection occurred among 11 Picazuro pigeons (Patagioenas picazuro) housed in an aviary with other bird species in the Netherlands. The birds lost weight, had ruffled feathers, and were anorexic. Despite treatment with fluids, force-feeding, and in 1 bird, doxycycline treatment $(50 \mathrm{mg} / \mathrm{kg} 1 \times / \mathrm{d})$, all 11 animals died or were euthanized. Necropsy revealed that 9 of these birds were in poor physical condition, lacking fat and pectoral muscle mass. The livers and spleens were enlarged; the livers extended an average of $0.5 \mathrm{~cm}$ beyond the rear edge of the sternum, whereas the mean diameter of the spleens was $1.0 \mathrm{~cm}$, approximately twice as large as the normal size. We suspected Chlamydia infection because of intracellular inclusions in Stamp (modified Ziehl Neelsen)-stained cytology of liver and spleen. We found multifocal heterophilic and lymphoplasmacytic infiltrates with necrosis in the liver and lymphoid depletion with necrosis and heterophilic infiltrates in the spleen. We stained slides with polyclonal antibodies against Chlamydia (bioMérieux, https://www.biomerieux.com) after a standard Avidin Biotin Complex protocol (4); liver and kidney tissues from 7 birds tested positive for Chlamydia. We did not observe any histologic changes consistent with viral inclusions or bacterial infection.

Because psittacosis in birds is a notifiable disease in the Netherlands, we informed public health authorities of our results. We forwarded frozen tissue samples to the Wageningen Bioveterinary Research institute to confirm C. psittaci infection. We also collected and forwarded 2 Picazuro pigeon carcasses and 3 pooled fecal samples from contact birds (i.e., Roseate spoonbill [Platalea ajaja], Puna ibis [Plegadis ridgwayi], and Scarlet ibis [Eudocimus ruber]), from the aviary. Two liver samples, 2 conjunctival and cloacal swabs, and 3 pooled fecal samples initially tested negative for C. psittaci, C. abortus, C. felis, and C. caviae in a PCR selective for the ompA gene. Because the liver and kidney samples of 7 pigeons tested positive for antibodies against Chlamydia, we submitted samples from all 11 pigeons and the 3 pooled fecal samples for further testing with real-time PCR selective for the 235 gene of Chlamydiaceae (5) and a duplex real-time PCR selective for C. gallinacea and C. avium $(3,6)$. All 11 pigeons tested positive for $C$. avium in $\geq 1$ samples of conjunctiva, cloaca, liver or intestines. The pooled fecal samples of contact birds tested negative in a PCR for Chlamydiaceae (Appendix, https://wwwnc. cdc.gov/EID/article/26/10/20-0086-App1.pdf). 